\title{
Analytical Formulations in the Domain Decomposition Method using Cylindrical Control Surface
}

\author{
Bo Tian , Hui-long Ren \\ College of ShipBuliding Engineering, Harbin Engineering University, Harbin, Heilongjiang, China
}

\begin{abstract}
The seakeeping problem is very important for marine structures, but present boundary element methods have still unresolved difficulties. In this work, the Green function method (Green function satisfying the linear boundary condition at free surface) is combined with the Rankine panel method so that two methods keep their advantages and brings important benefits. The fluid domain is divided into two subdomains by a control surface of cylindrical form. In the interior one the ship is arbitrary, the Rankine source method is then applied. In the exterior one the shape of the control surface is analytically known while the velocity potential and its normal derivative are expressed by expansions of orthogonal functions. Across the control surface two additional conditions must be satisfied: both the velocity potential and its normal derivative are to be continuous. In order to improve the computational accuracy, velocity potential is decomposed by using the Fourier series in polar direction and Laguerre function in vertical direction on the cylindrical control surface. The matrix elements of boundary integral equations consist of analytical integrations in wavenumber. Furthermore, the numerical results of the integrals are presented for seakeeping problems.
\end{abstract}

\section{Introduction}

The Rankine panel method is one of the well-known methods used for sea-keeping problems. It uses the simple Green function $(1 / r)$ for unbounded fluid, where $r$ is the distance between the field point and the source point. On the other hand, to satisfy the free surface and body boundary conditions, the integrations must be performed over all surfaces. Thus, a large amount of panels are necessary due to panelizing the free surface. In addition, to avoid the reflected wave from the sides of a numerical fluid domain a damping zone has to be introduced [1], [2].

As it is known, the implementation of the Kelvin source method using the Green function for a moving and oscillating source arises to several well-known problems concerning its wave component. In particular, it behaves with singularities and high oscillations when both the field and source points tend to the free surface. Also, it is the slow convergent integral defined along the dispersion curves. In every case, for each body shape this wave component should be evaluated very carefully [1], [2].

In this paper, we modify the Green function method (Green function satisfies the free surface boundary condition) by combining it with the Rankine panel method. In this work, we suggest to divide the fluid domain into two subdomains by a control infinitely-long cylindrical surface. This surface separates the problem into two problems: the interior one in which the ship is of any form, the Green function is Rankine source Green function; the exterior one in which the shape of the control surface is known and velocity potential is assumed to be known. Across the control surface two additional conditions must be satisfied: both the velocity potential and its normal derivative are to be continuous. The second problem provides us the Dirichlet-Neumann map which is used to solve the first problem, and as result the original one by Rankine panel method.

The combination of these two methods keeps their advantages and brings important benefits: area to be discretized becomes smaller; no need to introduce the damping zone, the solution of the problem is that for unbound fluid domain. The calculation of the Green function for ship motion may be done only once for large set of the different ships for the one particular velocity $U$, incoming wave heading and frequency, and an additional parameter which describes the control surface.

\section{Problem statement}

A ship or a body of general shape floats in unbounded fluid. The fluid is assumed inviscid, incompressible and the flow is irrotational. The motions of the ship are assumed to be small so that the linear theory can be applied. The coordinate system is situated above the center of the mass of the ship on the level of the undisturbed free surface $(z=0)$, see Fig. 1 . The problem is described by the following boundary value problems for the velocity potential $\phi_{j}(x, y, z)[1]$. 


$$
\begin{array}{cc}
\Delta \phi_{j}(x, y, z)=0, & (x, y, z) \in \Omega \\
\frac{\partial}{\partial n} \phi_{j}(x, y, z)=n_{j}(x, y, z), & (x, y, z) \in B \\
\frac{\partial}{\partial z} \phi_{j}(x, y, z)-\frac{\omega^{2}}{g} \phi_{j}(x, y, z)=0, \quad(x, y, z) \in F
\end{array}
$$

where $\Omega$ is the fluid domain, $B$ is the submerged part of the body hull, $F$ is the free surface, $\vec{n}=\left(n_{1}, n_{2}, n_{3}\right)$ is the normal vector on the body directed toward the fluid, $\left(n_{1}, n_{2}, n_{3}\right)=\vec{r} \times \vec{n}, \vec{r}$ is a vector from a reference point to the point $(x, y, z)$ on the body, $g$ is acceleration due to the gravity, $\omega$ is an incoming wave frequency.

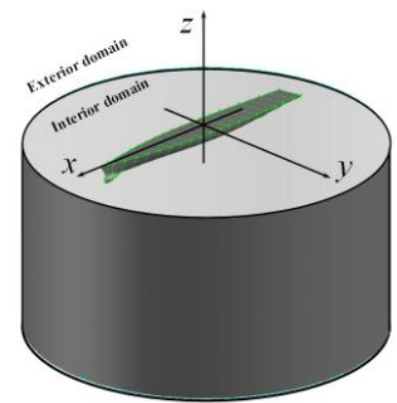

Figure 1. The coordinate system and the domain decomposition.

As it is shown in Fig.1, the cylindrical control surface $C$ divides the fluid domain into two sub-domains: the exterior domain $\Omega_{e}$ and the interior domain $\Omega_{i}$. The solution in the interior domain is solved using the constant Rankine panel method while in the exterior one Kelvin source method is applied. To couple both solutions two additional conditions should be used:

$$
\begin{gathered}
\phi_{j}^{e}(x, y, z)=\phi_{j}^{i}(x, y, z), \frac{\partial}{\partial n} \phi_{j}^{e}(x, y, z)=\frac{\partial}{\partial n} \phi_{j}^{i}(x, y, z), \\
(x, y, z) \in C
\end{gathered}
$$

which are the conditions of continuous of the velocity potential and its normal derivative across the control surface.

\section{Mathematical formulations}

\subsection{External Problem}

The cylindrical control surface is presented by distribution of singularities for the Green function is well known and is given as the following[1]:

$$
G(P, Q)=-\frac{1}{4 \pi r(P, Q)}-\frac{1}{4 \pi r^{*}(P, Q)}-\frac{k_{0}}{2 \pi} \int_{0}^{\infty} \frac{e^{-k|z+\zeta|}}{k-k_{0}} J_{0}(k R) d k
$$

where $k_{0}=\omega^{2} / g, g$ is acceleration due to the gravity, $\omega$ is an incoming wave frequency. $P(x, y, z)$ is a field point, $Q(\xi, \eta, \zeta)$ is a source point, $J_{0}$ is the Bessel function, $r(P, Q)$ and $r^{*}(P, Q)$ are the distances between the source and the field points and between the filed point and mirror of the source point with respect to the mean free surface $z=0, R^{2}=(x-\xi)^{2}+(y-\eta)^{2}$. This Green function satisfies the free surface condition.

Since the shape of control surface in this paper, it is convenient to introduce the cylindrical coordinate system in the following way and make the z-axis downwards in Fig. 2

$$
x=\rho \cos \psi, \quad y=\rho \sin \psi, \quad z=z
$$

here $\rho$ is a radius of the cylindrical surface which is given, $\psi \in[-\pi, \pi]$ varies in the plane $x O y$.

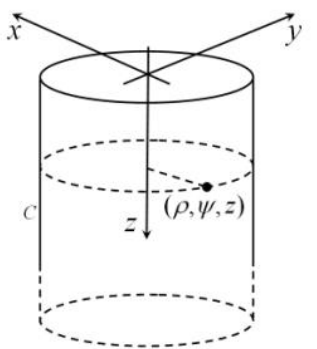

Figure 2. The control surface and cylindrical coordinate system.

Application of the Green's second identity provides

$$
\iint_{C}\left[\phi_{n}(Q) G(P, Q)-\phi(Q) G_{n}(P, Q)\right] d s=\left\{\begin{array}{cc}
0, & P \notin \Omega_{e} \\
\phi(P) / 2, & P \in \partial \Omega_{e} \\
\phi(P), & P \in \Omega_{e} \backslash \partial \Omega_{e}
\end{array}\right.
$$

here $n$ is the normal vector toward the fluid.

Any function of variables $\psi$ and $z$ can be presented in the form of infinite series using complete sets of base functions for each variable. For the convenience we chose harmonic functions $e^{i l \psi}$ and Laguerre functions $\ell_{k}(z)[3]$ :

$$
f(x, y, z)=\sum_{l=-\infty}^{\infty} \sum_{k=0}^{\infty} A_{k l} \ell_{k}(z) e^{i l \psi}
$$

where

$$
\ell_{k}(z)=e^{-\frac{z}{2}} L_{k}(z), \int_{0}^{\infty} \ell_{n}(z) \ell_{k}(z) d z=\int_{0}^{\infty} L_{n}(z) L_{k}(z) e^{-z} d z=\delta_{k n}
$$

here $L_{k}(z)$ is the Laguerre polynomials, $\delta_{k n}$ is the Kronecker delta. The coefficients $A_{k l}$ are found as[3]

$$
A_{k l}=\frac{1}{2 \pi} \int_{0}^{\infty} \int_{-\pi}^{\pi} f(x, y, z) \ell_{k}(z) e^{-i l \psi} d \psi d z, \quad(k, l) \in \mathbb{N} \times \mathbb{Z}
$$

Thus, according to (8)-(10), the velocity potential $\phi$ and its normal derivative $\varphi \equiv \phi_{n}$ can be rewritten in the form

$$
\phi(x, y, z)=\sum_{l=-\infty}^{\infty} \sum_{k=0}^{\infty} \phi_{k l} \ell_{k}(z) e^{i l \psi}, \quad \varphi(x, y, z)=\sum_{l=-\infty}^{\infty} \sum_{k=0}^{\infty} \varphi_{k l} \ell_{k}(z) e^{i l \psi}
$$

where the coefficients $\varphi_{k l}$ are unknown and $\phi_{k l}$ are assumed to be know.

After substituting (11) into (7), we obtain

$$
\begin{aligned}
\frac{1}{2} \sum_{l=-\infty}^{\infty} \sum_{k=0}^{\infty} \phi_{k l} \ell_{k}(z) e^{i l \psi} & =\iint_{C} G(P, Q)\left[\sum_{l=-\infty}^{\infty} \sum_{k=0}^{\infty} \varphi_{k l} \ell_{k}\left(z^{\prime}\right) e^{i l \psi^{\prime}}\right] d s \\
& -\iint_{C} G_{n}(P, Q)\left[\sum_{l=-\infty}^{\infty} \sum_{k=0}^{\infty} \phi_{k l} \ell_{k}\left(z^{\prime}\right) e^{i l \psi^{\prime}}\right] d s
\end{aligned}
$$

Here, we assumed that both points are on the same surface $C$ and we denoted $(\psi, z)=(x, y, z) \in C$ and $\left(\psi^{\prime}, z^{\prime}\right)=(\xi, \eta, \zeta) \in C$. Multiplying the both sides of (12) by $\frac{1}{2 \pi} \ell_{n}(z) e^{-i m \mu}$, then integrating the obtained product with respect to $(\psi, z) \in C[-\pi, \pi] \times[0,+\infty)$ yield 


$$
\begin{aligned}
\frac{1}{2} \phi_{n m} & =\frac{1}{2 \pi} \sum_{l=-\infty}^{\infty} \sum_{k=0}^{\infty} \varphi_{k l} \int_{0}^{\infty} \int_{-\pi}^{\pi} \iint_{C} G(P, Q) \ell_{k}\left(z^{\prime}\right) e^{i l \psi^{\prime}} \ell_{n}(z) e^{-i m \psi} d s d \psi d z \\
& -\frac{1}{2 \pi} \sum_{l=-\infty}^{\infty} \sum_{k=0}^{\infty} \phi_{k l} \int_{0}^{\infty} \int_{-\pi}^{\pi} \iint_{C} G_{n}(P, Q) \ell_{k}\left(z^{\prime}\right) e^{i l \psi^{\prime}} \ell_{n}(z) e^{-i m \psi} d s d \psi d z
\end{aligned}
$$

As it is shown in Fig.3, the integral infinitesimal of the control surface

$$
d s=d l d z=\rho d \psi d z
$$

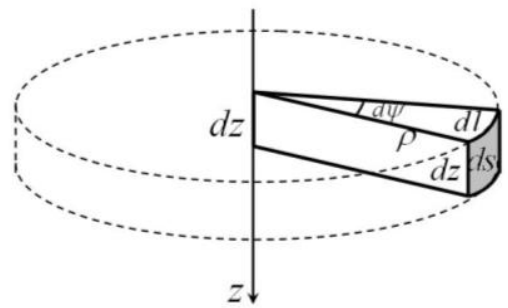

Figure 3. The integral infinitesimal.

hence,

$\iint_{C} f(r, \psi, z) d s=\iint_{r=\rho} f(\rho, \psi, z) d s=\int_{0}^{+\infty} \int_{-\pi}^{\pi} f(\psi, z) \rho d \psi d z$

then, (13) is

$$
\begin{aligned}
\frac{1}{2} \phi_{m n} & \frac{\rho}{2 \pi} \sum_{l=-\infty}^{\infty} \sum_{k=0}^{\infty} \varphi_{k} \int_{0}^{\infty} \int_{-\pi}^{\pi} \int_{0}^{\infty} \int_{-\pi}^{\pi} G(P, Q) \ell_{k}\left(z^{\prime}\right) e^{i l \psi^{\prime}} \ell_{n}(z) e^{-i m \psi} d \psi^{\prime} d z^{\prime} d \psi d z \\
& -\frac{\rho}{2 \pi} \sum_{l=-\infty}^{\infty} \sum_{k=0}^{\infty} \phi_{k l} \int_{0}^{\infty} \int_{-\pi}^{\pi} \int_{0}^{\infty} \int_{-\pi}^{\pi} G_{n}(P, Q) \ell_{k}\left(z^{\prime}\right) e^{i l \psi^{\prime}} \ell_{n}(z) e^{-i m \psi} d \psi^{\prime} d z^{\prime} d \psi d z
\end{aligned}
$$

\subsection{Internal Problem}

Application of the Green's second identity provides

$$
\iint_{B+F+C}\left[\phi_{n}(Q) G(P, Q)-\phi(Q) G_{n}(P, Q)\right] d s=c(P) \phi(P), P \in B \cup F \cup C
$$

where

$$
c(P)=\left\{\begin{array}{l}
1 / 2, \quad P \in B \cup F \\
-1 / 2, \quad P \in C
\end{array}\right.
$$

and $n$ is a normal vector directed inward the fluid domain on the body B and free surface $\mathrm{F}$ and outward (that is why $c(P)=-1 / 2)$ on the control surface $C$,

$$
G(P, Q)=-\frac{1}{4 \pi r(P, Q)}
$$

is the Rankine Green function.

The both body hull and free surface are divided by and sub-surfaces (panels), respectively. Thus, (17) becomes

$$
\left(\sum_{\alpha=1}^{N_{B}} \iint_{B^{a}}+\sum_{\beta=1}^{N_{E}} \iint_{F^{\natural}}+\iint_{C}\right)\left[\phi_{n}(Q) G(P, Q)-\phi(Q) G_{n}(P, Q)\right] d s=c(P) \phi(P)
$$

According to the boundary conditions, the normal derivative on the body is known function and on the free surface, due to the free surface boundary condition (3), it is

$$
\frac{\partial \phi}{\partial n}=-\frac{\partial \phi}{\partial z}=-k \phi, \quad z=0,
$$

Hence, (20) can be rewritten as the following

$$
\begin{aligned}
c(P) \phi(P) & =\iint_{C}\left[\phi_{n}(Q) G(P, Q)-\phi(Q) G_{n}(P, Q)\right] d s \\
& +\sum_{\alpha=1}^{N_{B}} \iint_{B^{\alpha}} \phi_{n}(Q) G(P, Q) d s-\sum_{\alpha=1}^{N_{B}} \iint_{B^{\alpha}} \phi(Q) G_{n}(P, Q) d s \\
& -\sum_{\beta=1}^{N_{F}} \iint_{F^{\beta}} \phi(Q)\left[k G(P, Q)+G_{n}(P, Q)\right] d s
\end{aligned}
$$

On each panel the velocity potential and its normal derivative are assumed to be a constant and we may write

$$
\begin{aligned}
c(P) \phi(P) & =\iint_{C^{\prime}}\left[\phi_{n}(Q) G(P, Q)-\phi(Q) G_{n}(P, Q)\right] d s \\
& +\sum_{\alpha=1}^{N_{B}} \varphi_{\alpha} \iint_{B^{a}} G(P, Q) d s-\sum_{\alpha=1}^{N_{B}} \phi_{\alpha} \iint_{B^{a}} G_{n}(P, Q) d s \\
& -\sum_{\beta=1}^{N_{F}} \phi_{\beta} \iint_{F^{\beta}}\left[k G(P, Q)+G_{n}(P, Q)\right] d s
\end{aligned}
$$

On the surface $C$ the velocity potential and its normal derivative are defined as:

$$
\phi(\xi, \eta, \zeta)=\sum_{l=-\infty}^{\infty} \sum_{k=0}^{\infty} \phi_{k l} \ell_{k}\left(z^{\prime}\right) e^{i l \psi^{\prime}}, \quad \varphi(\xi, \eta, \zeta)=\sum_{l=-\infty}^{\infty} \sum_{k=0}^{\infty} \varphi_{k l} \ell_{k}\left(z^{\prime}\right) e^{i l \psi^{\prime}}
$$

The substitution (24) into (23) yields

$$
\begin{aligned}
c(P) \phi(P) & =\sum_{l=-\infty}^{\infty} \sum_{k=0}^{\infty} \varphi_{k l} \iint_{C^{\prime}} \ell_{k}\left(z^{\prime}\right) e^{i l \psi^{\prime}} G(P, Q) d s \\
& -\sum_{l=-\infty}^{\infty} \sum_{k=0}^{\infty} \phi_{k l} \iint_{C} \ell_{k}\left(z^{\prime}\right) e^{i l \psi^{\prime}} G_{n}(P, Q) d s \\
& +\sum_{\alpha=1}^{N_{B}} \varphi_{\alpha} \iint_{B^{\alpha}} G(P, Q) d s-\sum_{\alpha=1}^{N_{B}} \phi_{\alpha} \iint_{B^{\alpha}} G_{n}(P, Q) d s \\
& -\sum_{\beta=1}^{N_{F}} \phi_{\beta} \iint_{F^{\beta}}\left[k G(P, Q)+G_{n}(P, Q)\right] d s
\end{aligned}
$$

Depending on the position of the point $P$, different cases should be considered:

When $P \in C$, According to (11), we can get

$$
\begin{aligned}
-\frac{1}{2} \phi_{n m} & =\sum_{l=-\infty}^{\infty} \sum_{k=0}^{\infty} \varphi_{k l} \frac{1}{2 \pi} \int_{0}^{\infty} \int_{-\pi}^{\pi} \iint_{C^{\prime}} \ell_{k}\left(z^{\prime}\right) e^{i l \psi^{\prime}} G(P, Q) \ell_{n}(z) e^{-i m \psi} d s d \psi d z \\
& -\sum_{l=-\infty}^{\infty} \sum_{k=0}^{\infty} \phi_{k l} \frac{1}{2 \pi} \int_{0}^{\infty} \int_{-\pi}^{\pi} \iint_{C} \ell_{k}\left(z^{\prime}\right) e^{i \psi^{\prime}} G_{n}(P, Q) \ell_{n}(z) e^{-i m \psi} d s d \psi d z \\
& +\sum_{\alpha=1}^{N_{B}} \varphi_{\alpha} \frac{1}{2 \pi} \int_{0}^{\infty} \int_{-\pi}^{\pi} \iint_{B^{G}} G(P, Q) \ell_{n}(z) e^{-i m \psi} d s d \psi d z \\
& -\sum_{\alpha=1}^{N_{B}} \phi_{\alpha} \frac{1}{2 \pi} \int_{0}^{\infty} \int_{-\pi}^{\pi} \iint_{B^{a}} G_{n}(P, Q) \ell_{n}(z) e^{-i m \psi} d s d \psi d z \\
& -\sum_{\beta=1}^{N_{F}} \phi_{\beta} \frac{1}{2 \pi} \int_{0}^{\infty} \int_{-\pi}^{\pi} \iint_{F^{P}}\left[k G(P, Q)+G_{n}(P, Q)\right] \ell_{n}(z) e^{-i m \psi} d s d \psi d z
\end{aligned}
$$

When $P_{\gamma}=P \in B^{\gamma}$ or $P_{\gamma}=P \in F^{\gamma}$, where $\gamma$ is the number of the panel, then (25) becomes

$$
\begin{aligned}
c\left(P_{\gamma}\right) \phi_{\gamma} & =\sum_{l=-\infty}^{\infty} \sum_{k=0}^{\infty} \varphi_{k l} \iint_{C^{\prime}} \ell_{k}\left(z^{\prime}\right) e^{i l \psi^{\prime}} G\left(P_{\gamma}, Q\right) d s \\
& -\sum_{l=-\infty}^{\infty} \sum_{k=0}^{\infty} \phi_{k l} \iint_{C^{\prime}} \ell_{k}\left(z^{\prime}\right) e^{i l \psi^{\prime}} G_{n}\left(P_{\gamma}, Q\right) d s \\
& +\sum_{\alpha=1}^{N_{B}} \varphi_{\alpha} \iint_{B^{a}} G\left(P_{\gamma}, Q\right) d s-\sum_{\alpha=1}^{N_{B}} \phi_{\alpha} \iint_{B^{a}} G_{n}\left(P_{\gamma}, Q\right) d s \\
& -\sum_{\beta=1}^{N_{F}} \phi_{\beta} \iint_{F^{\beta}}\left[k G\left(P_{\gamma}, Q\right)+G_{n}\left(P_{\gamma}, Q\right)\right] d s
\end{aligned}
$$

(26) and (27) from the system of linear algebraic equations, which in the matrix form can be written as 


$$
\mathbf{M \Phi}=\mathbf{F}, \quad \boldsymbol{\Phi}=\left(\phi_{\alpha}, \phi_{\beta}, \phi_{k l}\right)^{T} \quad \mathbf{F}=\left(F_{1}, F_{2}, F_{3}\right)^{T}
$$

where vector $\boldsymbol{\Phi}$ is unknown. The vector $\mathbf{F}$ is

$$
\begin{aligned}
& F_{1}=\left[\left\{\sum_{\alpha=1}^{N_{B}} \varphi_{\alpha} \iint_{B^{\alpha}} G\left(P_{\gamma}, Q\right) d s\right\}\right] \\
& F_{2}=\left[\left\{\sum_{\alpha=1}^{N_{B}} \varphi_{\alpha} \iint_{B^{\alpha}} G\left(P_{\gamma}, Q\right) d s\right\}\right] \\
& F_{3}=\left[\left\{\sum_{\alpha=1}^{N_{B}} \varphi_{\alpha} A_{B, n m, \alpha}\right\}\right]
\end{aligned}
$$

The structure of the matrix $\mathbf{M}$ is showed as follow:

$$
\mathbf{M}=\left[\begin{array}{lll}
M_{11} & M_{12} & M_{13} \\
M_{21} & M_{22} & M_{23} \\
M_{31} & M_{32} & M_{33}
\end{array}\right]=\left[\begin{array}{lll}
B \leftrightarrow B & B \leftrightarrow F & B \leftrightarrow C \\
F \leftrightarrow B & F \leftrightarrow F & F \leftrightarrow C \\
C \leftrightarrow B & C \leftrightarrow F & C \leftrightarrow C
\end{array}\right]
$$

\section{Numerical results}

In order to calculate the see-keeping of a sphere as Fig. 4 showed, a hemisphere is calculated as Fig. 5 showed. Parameters of the calculation are given in Table 1. In numerical model all series are represented by the finite series

$$
\sum_{l=-\infty}^{\infty} \sum_{k=0}^{\infty} \phi_{k l} \ell_{k}(z) e^{i l \psi} \rightarrow \sum_{l=-L_{\max }}^{L_{\max }} \sum_{k=0}^{K_{\max }} \phi_{k l} \ell_{k}(z) e^{i l \psi}
$$

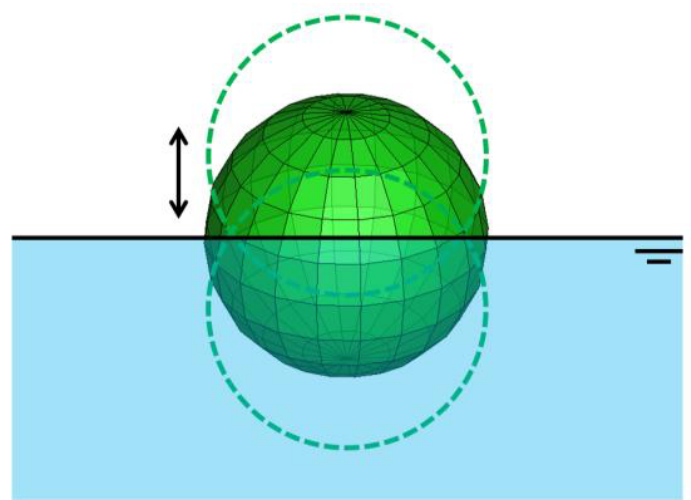

Figure 4. The heaving sphere.

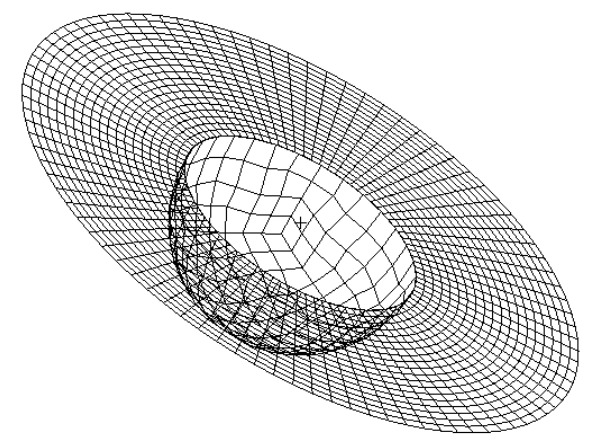

Figure 5. The floating sphere and the free-surface.

The results of velocity potential on the body panels with different frequency for comparing between the current method and the Kelvin source method are shown in the Fig. 6 to $11, \phi_{1}$ represents the velocity potential caused by the motion of surge, and $\phi_{3}$ represents the motion of heave.
Table 1. The parameters of floating sphere.

\begin{tabular}{|c|c|c|}
\hline Parameter's name & Notation & Values \\
\hline Radius & $r$ & $25 \mathrm{~m}$ \\
\hline Number of panels of the body & $N_{B}$ & 192 \\
\hline $\begin{array}{c}\text { Number of panels of the } \\
\text { free-surface }\end{array}$ & $N_{F}$ & 1710 \\
\hline Radius of the control surface & $R_{C}$ & $60 \mathrm{~m}$ \\
\hline Number of Laguerre functions & $K_{\max }$ & 10 \\
\hline Number of harmonic functions & $L_{\max }$ & 10 \\
\hline
\end{tabular}

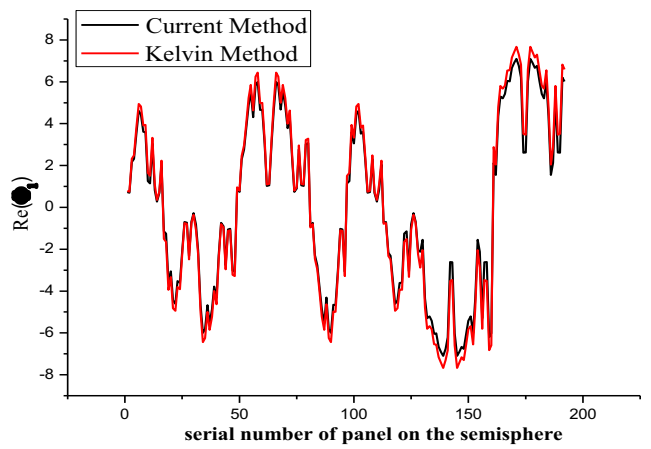

Figure 6. $\phi_{1}$ on each panel when the wave number $k=1.5$.

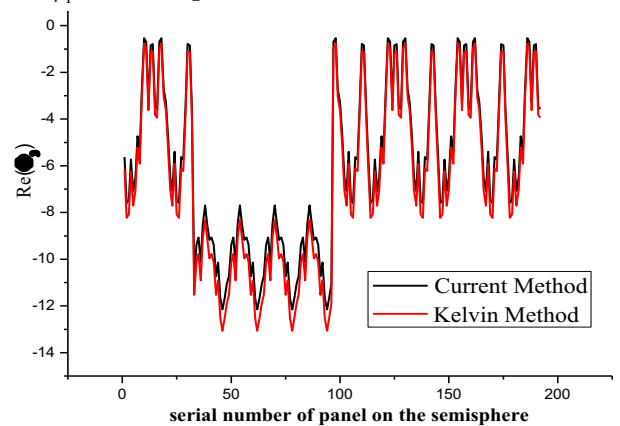

Figure 7. $\phi_{3}$ on each panel when the wave number $k=1.5$.

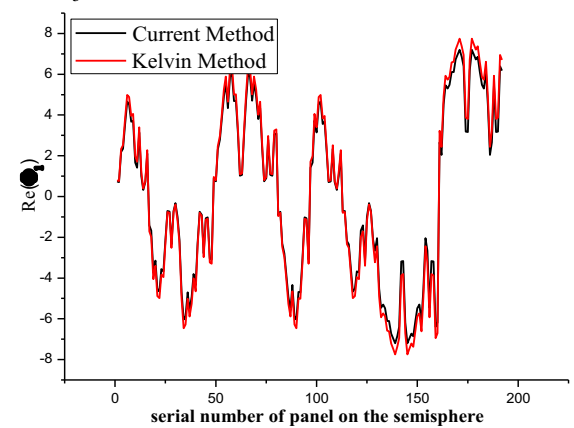

Figure 8. $\phi_{1}$ on each panel when the wave number $k=2.5$.

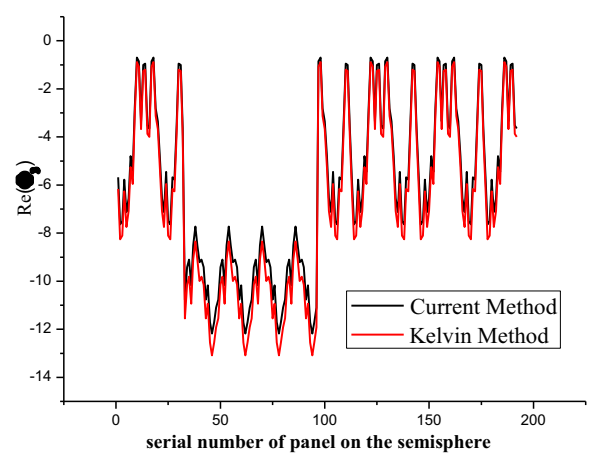

Figure 9. $\phi_{3}$ on each panel when the wave number $k=2.5$ 


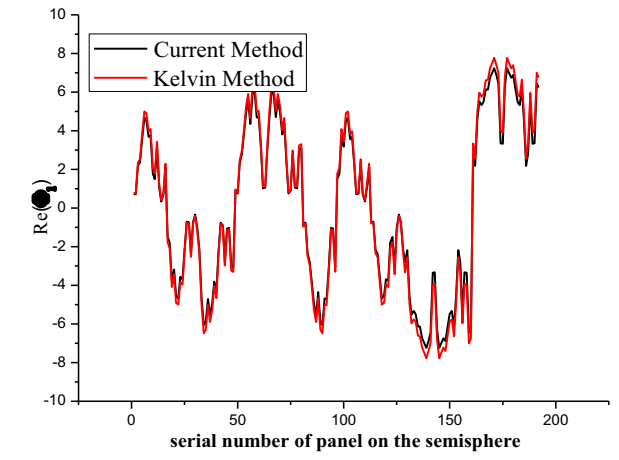

Figure 10. $\phi_{1}$ on each panel when the wave number $k=3.5$.

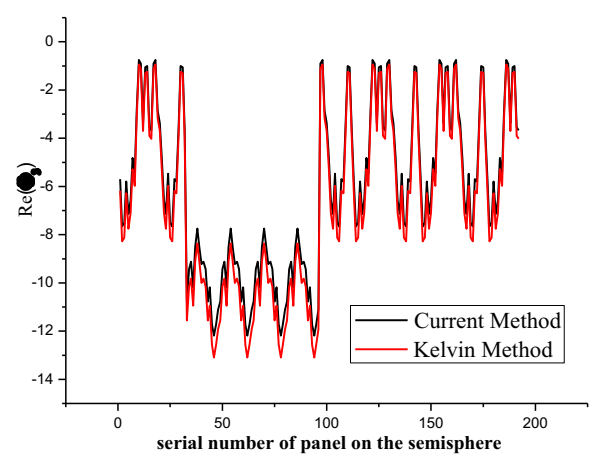

Figure 11. $\phi_{3}$ on each panel when the wave number $k=3.5$.

In order to study the effect of the control surface radius $R$ to calculation accuracy, this paper choses different $R / r$ values where the $r$ is the radius of hemisphere, the results are compared with the analytical values [4] as the Fig. 12 to Fig. 15 showed.

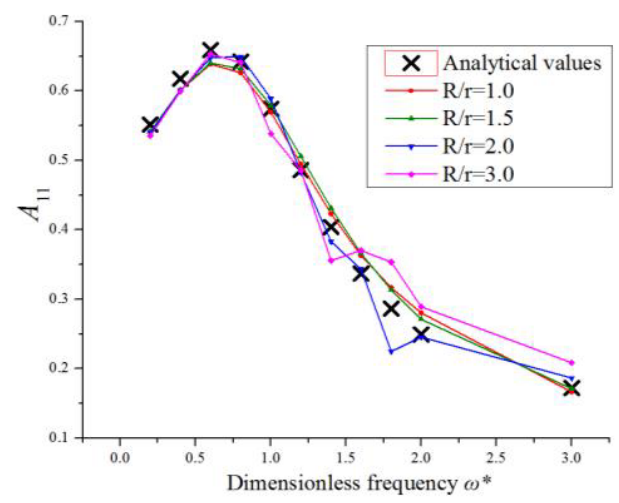

Figure 12. Surge added mass $A_{11}$ of hemisphere.

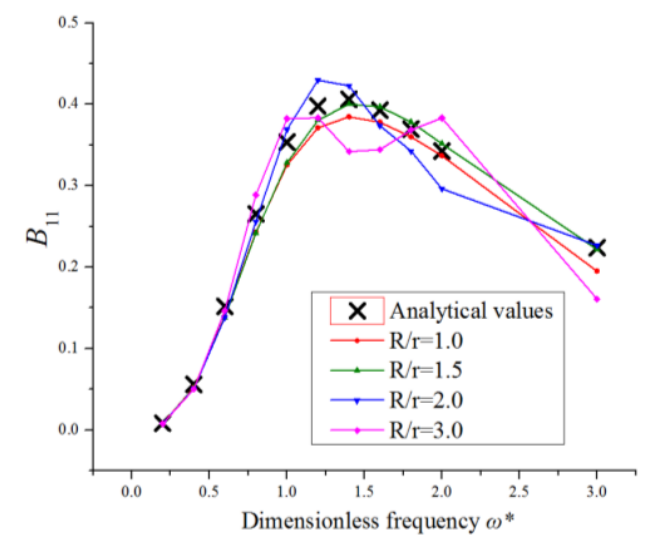

Figure 13. Surge damping coefficient $B_{11}$ of hemisphere.

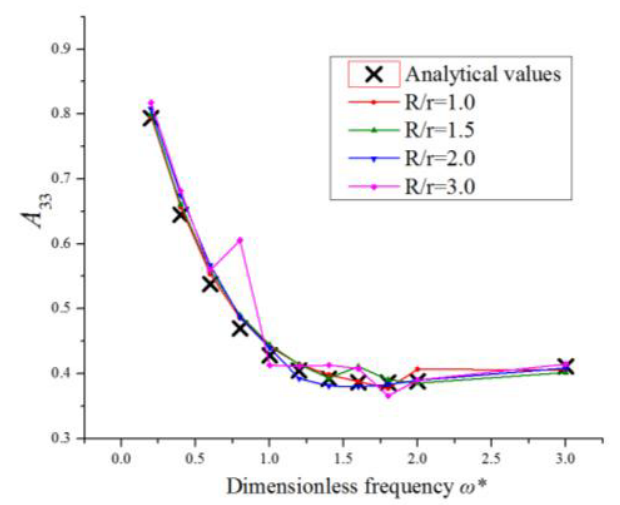

Figure 14. Heave added mass $A_{33}$ of hemisphere.

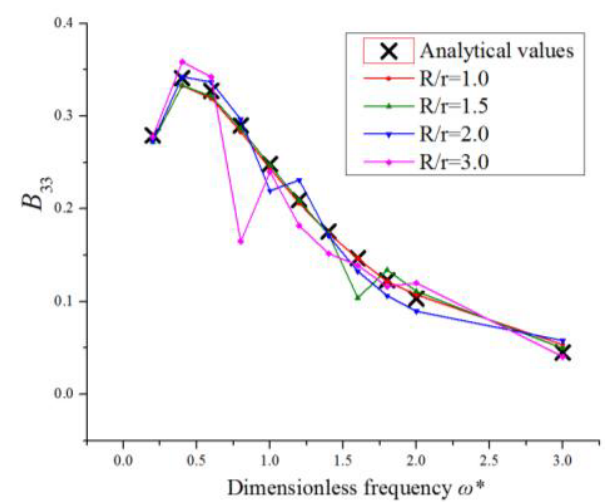

Figure 15. Heave damping coefficient $B_{33}$ of hemisphere.

The results show that with the control surface radius becoming larger, the deviation between numerical results and the analytical values of added mass and damping coefficient becomes larger. Therefore, choosing smaller control surface radius, not only ensures high accuracy, but also reduces the number of free-surface units to improve computational efficiency.

\section{Conclusions}

In this paper, we have considered the seakeeping problem for a hemisphere with zero speed using the hybrid method. The fluid domain is divided into two subdomains by a control surface of cylindrical form. In the interior one the Rankine source method is applied, and in the exterior one the Kelvin source method is applied. Unlike the classical BEM method, the control surface is not divided into panels, but uses the analytical expressions. Using the cylindrical surface, we can decouple the vertical and horizontal direction. The results of the comparison between the current method and the Kelvin method show that the velocity potentials on the panels of the ship fit well each other.

\section{References}

1. X.B.Chen, F.Dias and W.Y. Duan "Introduction of dissipation in potential flows," Proc. of 7th International Workshop on Ship Hydrodynamics, Shanghai, China, 213-322(2011)

2. I. Ten and X.B. Chen "A coupled Rankine-Green function method applied to the forward-speed 
seakeeping problem," Proc. 25th IWWWFB, Harbin, China, 165-168(2010)

3. A. Milton and A.S. Irene "Handbook of Mathematical functions, "Dover publications Inc, New York. (1964).

4. A.Hulme, "The wave forces acting on a floating hemisphere undergoing forced periodic oscillations", J. Fluid Mech., 121:443-463(1982)
5. OZDEMIR Y H, BARLAS B, YILMAZ T, BAYRAKTAR S. "Numerical and experimental study of turbulent free surface flow for a fast ship model". BRODOGRADNJA/SHIPBUILDING 65(1):39-54, (2014) 